
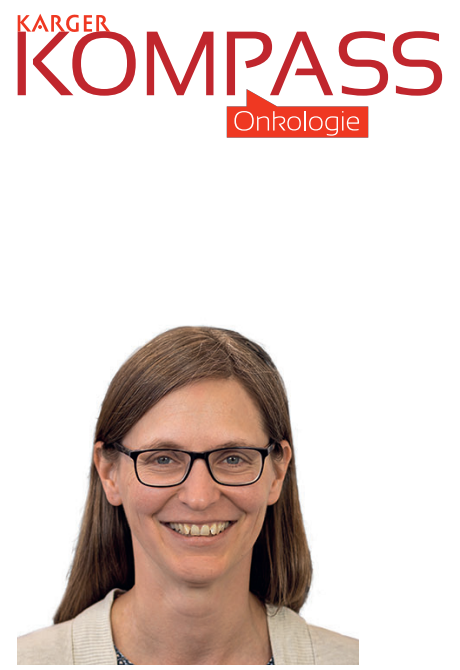

\section{Stefanie Dencks}

Lehrstuhl für Medizintechnik Fakultät für Elektrotechnik und Informationstechnik,

Ruhr-Universität Bochum,

Deutschland

\title{
Stefanie Dencks
}

\section{Trägerin des Innovationspreis der Stiftung Familie Klee 2019}

\section{Forschungsschwerpunkte}

- Signalanalyse im Ultraschall

- Bildgebung und Bildverarbeitung

- Verarbeitung stochastischer Signale

\section{Akademischer Werdegang}

- seit September 2011: Akademische Rätin am Lehrstuhl für Medizintechnik in der Fakultät für Elektrotechnik und Informationstechnik der Ruhr-Universität Bochum

- Februar 2009 - Juli 2011: Wissenschaftliche Angestellte im Fachbereich Schall, Arbeitsgruppe Ultraschall der Physikalisch-Technischen Bundesanstalt, Braunschweig; Themenschwerpunkt: hochintensiver fokussierter therapeutischer Ultraschall

- Juli 2009: Abschluss der Promotion «Signal Analysis of Quantitative Ultrasound Measurements at the Proximal Femur» mit Auszeichnung

- März 2003 - Oktober 2007: Wissenschaftliche Angestellte in der Medizinischen Physik, Diagnostische Radiologie des Universitätsklinikum Schleswig-Holstein

- 1996 - 2002: Studium der Elektrotechnik an der Universität Hannover und an der Technischen Universität Dresden

Frau Dr. Dencks, hatten Sie schon immer einen besonderen Blick für Details?

Dr. Stefanie Dencks: Ich denke schon. Ohne Faszination für das Detail ist Forschung wahrscheinlich sowieso schwierig. Aber es stimmt schon, dass es mich immer besonders interessiert hat, wenn es in Projekten um sehr kleine Ströme oder versteckte Signale ging oder auch im Ästhetischen um die perfekte Darstellung. Grundsätzlich liegt es mir, auf Details zu achten.

In Ihrer Forschungsarbeit, die im Juni von der Stiftung Familie Klee mit dem Innovationspreis ausgezeichnete wurde, nutzen Sie winzige Bläschen, um Blutgefäße von Tumoren sichtbar zu machen. Woher kommt die Idee dazu?

Dr. Dencks: Die Idee, einzelne Mikrobläschen zu lokalisieren und damit Bilder mit höherer Auflösung zu erzeugen, ist unabhängig voneinander in einer französischen Gruppe und in unserer Gruppe entstanden. Während die französische Gruppe durch entsprechende optische Methoden der Lokalisationsmikroskopie (FPALM) motiviert wurde, war es bei uns eine fast zufällige Entdeckung. Wir wollten eigentlich zur Quantisierung einzelne Mikrobläschen zählen und hatten dazu ihre lokalisierten Mittelpunkte farbig eingezeichnet. Der nächste Schritt, die Mikrobläschen über die Zeit zu verfolgen und Gefäßbäume zu erzeugen, lag dann nah. Durch den starken klinischen Bezug über unseren langjährigen Ko-

\section{KARGER}

Fax +497614520714 information@karger.com www.karger.com (c) 2019 S. Karger GmbH, Freiburg
Dr.-Ing. Stefanie Dencks

Lehrstuhl für Medizintechnik

Fakultät für Elektrotechnik und Informationstechnik

Ruhr-Universität Bochum

Universitätsstraße 150, 44801 Bochum, Deutschland

Stefanie.dencks@rub.de 


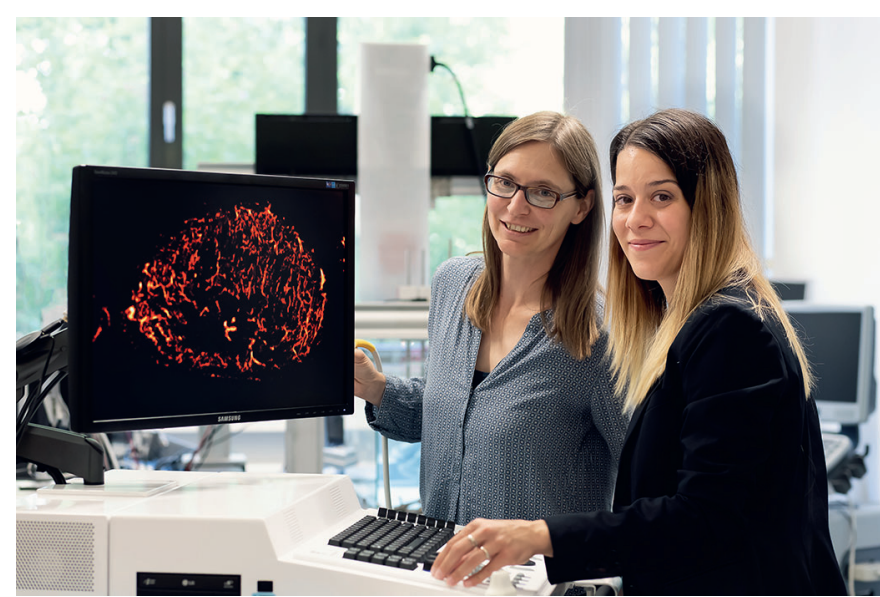

Dr. Stefanie Dencks (links) und Dr. Tatjana Opacic vom Institut für Experimentelle Molekulare Bildgebung der Uniklinik der RheinischWestfälischen Technischen Hochschule (RWTH) Aachen wurden mit dem Innovationspreis der Stiftung Familie Klee ausgezeichnet.

operationspartner Prof. Fabian Kiessling (EXMI, RWTH Aachen) konnten wir das Verfahren dann als erste im lebenden Organismus und später auch erstmals klinisch einsetzen.

\section{Welches ist die zentrale Erkenntnis Ihrer Forschung?}

Dr. Dencks: Die zentrale Erkenntnis ist, dass mit Hilfe der ULM selbst kleinste Gefäßstrukturen und deren Durchblutung dargestellt werden können und das auch - wenn auch derzeit noch mit relativ hohem Aufwand - in klinischen Anwendungen. Noch ist es etwas früh, aber es scheint, dass wir dadurch auch neue Erkenntnisse über das Wachstum von Tumoren und deren Reaktionen auf Therapien gewinnen.

\section{Worin liegt die größte Schwierigkeit in Ihrer Forschung?}

Dr. Dencks: Nachdem ULM prinzipiell funktioniert und viele der einzelnen Arbeitsschritte optimiert wurden, besteht die größte Schwierigkeit darin, geeignete Messprotokolle für die klinische Anwendung und eine automatisierte Auswertung der US-Sequenzen zu entwickeln. Häufig bleibt in der Klinik nicht genug Zeit, um auf die Abbildung des gesamten Gefäßbaums zu warten, der ja an allen Stellen erst von Mikrobläschen passiert werden muss. Hier entwickeln wir aktuell statistische Verfahren, um trotzdem aussagekräftige Parameter mit klinisch realisierbaren Messzeiten berechnen zu können.
Wie können Ihre Forschungsergebnisse Einfluss auf die Behandlungspraxis haben?

Dr. Dencks: Die Einflüsse können sehr vielfältig sein. Letztlich geht es bei vielen Erkrankungen um die Charakterisierung von Gefäßneubildung. In Bezug auf Tumore bedeutet das z.B. einerseits, dass eine Therapie überwacht und individuell angepasst werden kann. Andererseits ermöglicht ULM auch prinzipielle Erkenntnisse über Wirkprozesse, so dass Therapien weiterentwickelt oder verschiedene Tumorarten anhand ihrer charakteristischen Gefäßneubildung unterschieden werden können. Dies ist übertragbar auf alle Pathologien, in denen Angiogenese oder die Struktur kleiner Gefäße eine Rolle spielt.

Welchen Fragen wollen Sie als nächstes auf den Grund gehen?

Dr. Dencks: Die nächste große Herausforderung und Chance für das ULM-Verfahren ist die Erweiterung auf 3D. Hierbei fallen große Datenmengen an und die Verfolgung vieler einzelner Bläschen wird für die Algorithmen komplexer. Andererseits ermöglicht dies die Abbildung vollständiger Gefäßbäume und die korrekte Bestimmung von Geschwindigkeiten in drei Dimensionen.

Darüber hinaus interessiert uns, wie wir aus den reinen Abbildungen klinisch aussagekräftige Parameter definieren und bestimmen können. Dabei sollen dann auch Techniken des Deep Learning eingesetzt werden. Hierbei sind wir immer auf den engen Austausch mit unseren klinischen Partnern angewiesen.

Wenn Sie sich etwas wünschen könnten für Ihre Forschung, was wäre das?

Dr. Dencks: Auf der technischen Seite würde ich mir ein klinisch zugelassenes 3D-Ultraschallgerät wünschen, mit dem ich die neuen dreidimensionalen Methoden auch unmittelbar zur klinischen Anwendung bringen könnte. Wir möchten hier möglichst schnell vorwärtskommen, sind aber auf entsprechende Förderung angewiesen und für Projekte, die aus der Grundlagenforschung kommend ein neues Verfahren für die klinische Anwendung entwickeln, ist die Förderlandschaft in Deutschland nicht ganz einfach. Für ein Feld, dass sich international so dynamisch entwickelt wie ULM, sind die Entscheidungszeiträume vieler Förderer viel zu lang und die Laufzeiten der Projekte dann wiederum zu kurz.

Frau Dr. Dencks, wir danken Ihnen herzlich für das Gespräch und wünschen weiterhin viel Erfolg! 